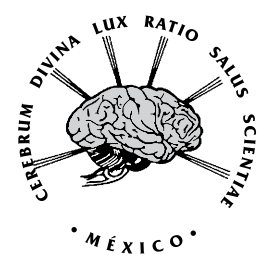

\title{
Epilepsia de ausencia juvenil. Evaluación neuropsicológica de dos casos
}

\author{
Absence epilepsy in young people. \\ Neuropsychological evaluation of two cases
}

\author{
Amador Barreda Guzmán, * Dora Elizabeth Granados Ramos*
}

* Universidad Veracruzana, Facultad de Psicología-Xalapa, Laboratorio de Psicobiología.

\begin{tabular}{|c|c|}
\hline $\begin{array}{r}\text { Palabras clave: } \\
\text { Epilepsia, crisis } \\
\text { de ausencia, } \\
\text { evaluación } \\
\text { neuropsicológica. }\end{array}$ & $\begin{array}{l}\text { Introducción: Las epilepsias constituyen trastornos de la función neuronal expresados en crisis de episodios limitados causadas por } \\
\text { descargas anormales de grupos de neuronas. La epilepsia de ausencia juvenil inicia entre los } 10 \text { y } 17 \text { años, con ausencias típicas } \\
\text { y en ocasiones crisis tónico-clónicas. Ambos sexos son igualmente afectados. El electroencefalograma (EEG) intercrítico presenta } \\
\text { descargas de punta-onda de } 3 \mathrm{~Hz} \text { con buena respuesta al medicamento. Las evaluaciones neuropsicológicas permiten determinar } \\
\text { la presencia de cambios cognoscitivos y comportamentales. Material y métodos: Estudio observacional, descriptivo, transversal, } \\
\text { prospectivo. Se evaluaron dos estudiantes de la Licenciatura en Psicología (casos A y B), de } 19 \text { años de edad, de lateralidad diestra, } \\
\text { sexo femenino. En ambos casos se reportaron crisis de ausencia con inicio en la adolescencia; el caso B presentaba además crisis } \\
\text { tónico-clónicas. Ambas se encontraban tomando medicamento en el momento de la evaluación. En forma individual, en un cubículo } \\
\text { bien iluminado, libre de distractores, se aplicaron en dos sesiones de } 45 \text { minutos en promedio la evaluación neuropsicológica breve } \\
\text { en español (NEUROPSI), el test de clasificación de Wisconsin versión computarizada (WISCONPC) y la escala de somnolencia } \\
\text { Epworth. Resultados: En NEUROPSI, el caso A, obtuvo puntaje total de } 118 \text { (normal alto) y el caso B, } 106 \text { (normal) con dificultades } \\
\text { en atención, evocación de estímulos verbales y comprensión lectora. En WISCONPC, el caso A completó seis categorías, mientras } \\
\text { que el B completó dos, presentando incapacidad para mantener la organización. En la escala Epworth, el caso B reportó no dormir } \\
\text { suficiente. Discusión y conclusiones: Las evaluaciones neuropsicológicas nos permitieron conocer el estado actual de los procesos } \\
\text { cognoscitivos de los casos evaluados e indicar estrategias de corrección en los procesos observados con dificultades. }\end{array}$ \\
\hline
\end{tabular}

\section{ABSTRACT}

Key words:

Epilepsy, absence seizures, neuropshychologycal assessment.
Introduction: Epilepsy constitutes disorders of neuronal function expressed as limited crisis episodes caused by abnormal discharges of groups of neurons. Juvenil absence epilepsy begins between 10 and 17 years old, with typical absences and sometimes tonic-clonic seizures. Intecritical electroencephalogram (EEG) presents $3 \mathrm{~Hz}$ spike-and-wave discharges with a good response to medication. Neuropsychological assessments allow determining the presence of cognitive and behavioral changes. Material and methods: Observational, descriptive, transversal and prospective research. We evaluated two righthanded female, 19 years old, Psychology undergraduate students (Subjects $A$ and B). Both subjects reported absence seizures beginning in adolescence, subject $B$ also reported tonic-clonic seizures. In a well illuminated room, free of distractors, in an individual manner, we applied the brief neuropsychological test in spanish (NEUROPSI), the Wisconsin card sorting test computerized version (WISCONPC) and the Epworth Sleepiness Scale in two 45-minutes sessions. Results: In the NEUROPSI, subject $A$, got a total score of 118 (high normal) and subject $B$ a score of 106 (normal) with difficulties in attention, recall of verbal stimuli and reading comprehension. In the WISCONPC, subject A, completed six categories while B completed two categories, with an inability to keep the organization. In the Epworth Scale, subject B reported not sleeping enough. Discussion and conclusions: Neuropshychologycal assessments allowed us to know the current condition of cognitive processes of evaluated subjects and to suggest corrective strategies in the identified processes with difficulties.

Recibido para publicación: 18/03/2014. Aceptado: 11/08/2014.

Este artículo puede ser consultado en versión completa en: www.medigraphic.com/enfermerianeurologica 


\section{PLANTEAMIENTO DEL PROBLEMA}

Las epilepsias son alteraciones del sistema nervioso caracterizadas por la presencia de crisis convulsivas recurrentes y espontáneas producto de la sincronización anormal de un grupo de neuronas. En el mundo existen alrededor de 50 millones de personas que padecen epilepsia, de las cuales cinco millones viven en América Latina. ${ }^{1}$ En México se estima que entre el 1.8 y $2 \%$ de la población presenta epilepsia, lo cual representa más de un millón de pacientes y al menos el $76 \%$ de estos casos inician con crisis en la adolescencia. ${ }^{2}$

Las personas que padecen epilepsia pueden presentar deterioro cognoscitivo, que varía según la edad de aparición, la frecuencia y tipo de crisis y la medicación prescrita; las disfunciones en las capacidades cognoscitivas del paciente con epilepsia interfieren en su desarrollo personal, académico, laboral y social.

Las crisis convulsivas que se desarrollan a partir de los 18 años, consideradas tardías, suelen provocar alteraciones en el rendimiento neuropsicológico.

Las dificultades presentes en los pacientes con epilepsia se identifican mediante evaluaciones neuropsicológicas, las cuales caracterizan el perfil neuropsicológico, ubicándolas en áreas fuertes y débiles.

En los casos con epilepsia, se han descrito dificultades en las áreas de atención, memoria y funciones ejecutivas que pueden interferir tanto en la vida diaria como en las actividades académicas.

La identificación de dificultades en los casos con epilepsia mediante la utilización de instrumentos validados permitirá generar estrategias de estimulación cognoscitiva que favorezcan el desarrollo integral del paciente con epilepsia.

\section{INTRODUCCIÓN}

Las epilepsias, de acuerdo a la Liga Internacional contra la Epilepsia, ${ }^{3}$ se definen como una enfermedad cerebral cuando ocurren al menos dos crisis convulsivas no provocadas con espacio de 24 horas entre cada una o cuando existe probabilidad de presentarlas durante los 10 años siguientes tras dos crisis convulsivas no provocadas.

Las crisis epilépticas son alteraciones súbitas, involuntarias y limitadas en tiempo, manifestadas con cambios en la actividad motora o de la conciencia donde predomina la actividad eléctrica anormal. Se consideran de inicio temprano cuando aparecen antes de los 18 años de edad y tardías cuando lo hacen después de esa edad. ${ }^{4}$

De acuerdo a su etiología, ${ }^{5}$ las epilepsias se clasifican como:

a) Idiopáticas: en las cuales no existe lesión en el sistema nervioso y se asocia a factores genéticos.

b) Sintomáticas: asociadas a lesiones en el sistema nervioso que dieron origen al trastorno epiléptico.

c) Criptogénicas: se asume que es sintomática (puesto que existe lesión en el sistema nervioso) pero la causa no ha sido determinada. d) Provocadas: existen factores ambientales específicos que favorecen la aparición de crisis convulsivas. No existe daño estructural y algunas tienen base genética.

Las crisis epilépticas se clasifican en parciales o generalizadas. En las crisis parciales la actividad eléctrica anormal inicia y se mantiene en un área cerebral específica.

Las crisis parciales pueden ser simples (sin alteración de la conciencia) o complejas (con alteración de la conciencia).

En las crisis generalizadas, la descarga anormal de las neuronas afecta a toda la corteza cerebral y el sujeto pierde por completo la conciencia. ${ }^{6,7}$ Estas crisis se clasifican en:

a) Mioclónicas: producen contracciones involuntarias repentinas, duran menos de un segundo y ocurren en las extremidades inferiores o superiores del cuerpo.

b) Tónicas: producen aumento sostenido en la contracción muscular, tienen duración de dos a 10 segundos y ocasionalmente pueden dar lugar a una caída.

c) Clónicas: contracciones musculares repetitivas y sincrónicas que afectan al tono muscular de forma prolongada.

d) Tónico-clónicas: inician con aumento del tono muscular en la fase tónica, seguida de contracciones simétricas y sincrónicas en la fase clónica. También puede ocurrir primero la fase clónica y después la fase tónica.

e) Atónicas: producen disminución repentina del tono muscular; si el individuo se encuentra de pie, cae inevitablemente al suelo.

f) Ausencias: se presentan como pérdidas transitorias de la continuidad de la conciencia; el paciente interrumpe la actividad que está haciendo en ese momento y fija la vista al frente o puede girar los ojos hacia arriba; durante algunos segundos no responde, ni habla, ni atiende a los demás. Después de la crisis continúa lo que estaba haciendo y tal vez no se haya dado cuenta de ésta. Durante la crisis se detiene la actividad normal del cerebro por unos cuantos segundos y pueden haber leves movimientos involuntarios. Las ausencias pueden presentarse solas o estar asociadas con componentes tónicoclónicos.

La epilepsia de ausencia juvenil es una manifestación idiopática generalizada que inicia en la pubertad, consiste en crisis de ausencias que pueden expresarse con crisis mioclónicas y tónico-clónicas. Afecta a mujeres y a hombres de la misma forma y en algunos casos tiene antecedentes heredofamiliares de epilepsia.

En el electroencefalograma de estos casos se observa actividad basal normal con descargas de tipo punta-onda generalizada con frecuencia de $3 \mathrm{~Hz}$. La respuesta al tratamiento farmacológico es buena. ${ }^{8}$

Algunos estudios han demostrado que la epilepsia puede provocar alteraciones sobre el funcionamiento cerebral 
Barreda GA y cols. Epilepsia de ausencia juvenil

y a su vez condicionar la calidad de vida del paciente, interfiriendo en su desarrollo personal, académico, laboral y social. El deterioro neuropsicológico se ha asociado como comórbido en pacientes con epilepsia señalando deficiencias en las funciones cognoscitivas. ${ }^{9}$

Este deterioro funcional consecuente depende de diversos factores, como la frecuencia, la duración y el tipo de crisis, la etiología, la edad de inicio, los fármacos antiepilépticos administrados o la presencia de factores psicosociales difíciles. ${ }^{10}$

Las evaluaciones neuropsicológicas permiten determinar la presencia de cambios cognoscitivos y comportamentales, tanto en individuos sin dificultades cognoscitivas como en los que se sospecha algún tipo de disfunción cerebral. Estos cambios se definen y cuantifican mediante la observación clínica y la utilización de instrumentos de medición. ${ }^{11}$

La evaluación neuropsicológica en pacientes con epilepsia debe incluir la valoración de todas las áreas de la cognición: inteligencia, atención, resolución de problemas lógico-matemáticos, memoria, lenguaje, funciones ejecutivas, motoras y sensoriales. ${ }^{12}$

Los procesos psicológicos de atención y memoria se afectan con frecuencia en la epilepsia, sobre todo en casos que presentan crisis generalizadas; éstos muestran más dificultades en atención que aquellos que presentan solamente crisis parciales. ${ }^{13}$

En los pacientes con epilepsia también se han descrito alteraciones en los ciclos de sueño-vigilia, los cuales pueden relacionarse con las crisis epilépticas nocturnas o debido a la medicación antiepiléptica. ${ }^{14}$ Los malos hábitos de sueño también se relacionan con estas alteraciones.

El objetivo de la investigación consistió en caracterizar el perfil neuropsicológico de dos casos universitarios con epilepsia de ausencia juvenil.

\section{MATERIAL Y MÉTODOS}

Se realizó un estudio de tipo observacional, descriptivo, transversal y prospectivo con dos casos de sexo femenino (casos A y B), estudiantes de la Licenciatura en Psicología, de la Facultad de Psicología de la Universidad Veracruzana, Xalapa, Veracruz, México, con edad promedio de 19 años 5 meses y lateralidad diestra. En ambos casos se reportaron crisis de ausencia con inicio en la etapa juvenil; el caso $\mathrm{B}$, además, presentaba crisis tónico-clónicas. Ambos casos se encontraban medicados al momento de la evaluación (Cuadro I).

A las participantes se les explicó en qué consistían las evaluaciones y firmaron una carta de consentimiento informado.

En forma individual, en un cubículo bien iluminado, libre de distractores, se aplicaron en dos sesiones de 45 minutos en promedio la evaluación neuropsicológica breve en español (NEUROPSI), el test de clasificación de Wisconsin versión computarizada (WISCONPC) y la escala de somnolencia Epworth. Dos personas estandarizadas en las pruebas fueron las responsables de la evaluación.

\begin{tabular}{|c|c|c|}
\hline \multicolumn{3}{|c|}{ Cuadro I. Perfil general de los casos. } \\
\hline Datos & Caso A & Caso B \\
\hline Edad & 19 años 1 mes & 19 años 8 meses \\
\hline Años de estudio & 15 años & 16 años \\
\hline $\begin{array}{l}\text { Antecedentes here- } \\
\text { dofamiliares }\end{array}$ & $\begin{array}{l}\text { Prima paterna con } \\
\text { crisis de ausencia }\end{array}$ & Sin antecedentes \\
\hline $\begin{array}{l}\text { Edad de primera } \\
\text { crisis }\end{array}$ & 18 años 11 meses & 15 años 2 meses \\
\hline Crisis tónico-clónicas & Ausentes & Presentes \\
\hline Medicación & Fenitoína $(0.5-0-0)$ & Lamictal (1-0-0.5) \\
\hline $\begin{array}{l}\text { Fase del ciclo } \\
\text { menstrual }\end{array}$ & Folicular & Lútea \\
\hline
\end{tabular}

La evaluación neuropsicológica breve en español (NEUROPSI) ${ }^{15}$ es un instrumento breve que permite valorar los procesos cognoscitivos de orientación temporoespacial y en persona, atención y concentración, memoria (codificación y evocación), lenguaje, lectura, escritura y funciones ejecutivas. La prueba está estandarizada en población mexicana y tiene puntajes de corte de acuerdo a la edad y escolaridad del paciente. Los puntajes obtenidos en cada una de las subpruebas presentadas se contabilizaron para obtener el puntaje total, que se calificó como normal alto (>115), normal (103-114), leve (98-102), y severo $(<98)$ por rango de edad y años de estudio de las participantes evaluadas.

El test de clasificación de Wisconsin versión computarizada (WISCONPC) ${ }^{16}$ es una prueba neuropsicológica utilizada para evaluar las funciones cognoscitivas de conceptualización, categorización y flexibilidad cognoscitiva. La prueba inicia con la presentación de cuatro cartas muestra, con 128 cartas respuesta, las cuales son presentadas una por una para que el sujeto busque relación con alguna de las cartas muestra bajo una categoría, pudiendo ser color, forma o número. Se inicia con la categoría de color, después con forma y por último, número. La computadora retroalimenta al sujeto con las palabras "correcto", si lo hace bajo la categoría solicitada, o "incorrecto", si utiliza otro criterio de clasificación. Una vez terminada una categoría con diez respuestas correctas se pasa a la siguiente sin que se notifique al sujeto de este cambio. Cuando se terminan las tres categorías se repiten de nuevo en el orden de los criterios de color, forma y número. La prueba termina una vez que el sujeto completa las seis categorías o si se le acaban las 128 cartas-respuesta.

La escala de somnolencia Epworth ${ }^{17}$ es un instrumento autoaplicable de ocho reactivos que evalúa la tendencia a quedarse dormido en ocho situaciones diferentes. El sujeto responde cada reactivo en una escala del 0 al 3 , siendo 0 (nunca me quedaría dormido), 1 (ligera probabilidad de quedarme dormido), 2 (moderada probabilidad de quedarme dormido) o 3 (alta probabilidad de quedarme dormido). Los puntajes de cada reactivo se suman y proporcionan la calificación total, pudiendo resultar de 0 a 24, en donde de 
0 a 6 indica sueño suficiente, de 7 a 8 sueño promedio y de 9 a 24, sueño insuficiente.

A los casos se les entregaron los resultados de las evaluaciones por escrito.

\section{RESULTADOS}

En NEUROPSI el caso A obtuvo puntaje total de 118 (normal alto) y el caso B, 106 (normal); este último presentó dificultades en atención, evocación de estímulos verbales y comprensión lectora (Cuadro II).

En el WISCONPC (Cuadro III), el caso A formó seis categorías utilizando 83 estímulos, mientras que el caso B completó dos categorías ocupando los 128 estímulos disponibles; además presentó cinco incapacidades para mantener la organización, es decir, rompió el criterio de clasificación tras cinco respuestas correctas consecutivas.

En la escala Epworth (Cuadro IV), el caso A obtuvo un puntaje de 7, es decir, duerme dentro del promedio mientras que el caso B obtuvo 9, lo cual indica que no está durmiendo lo suficiente.

\section{DISCUSIÓN}

Los pacientes con epilepsia pueden presentar dificultades cognoscitivas que dependen de los problemas neuropsicológicos previos asociados y la medicación prescrita. ${ }^{18}$

Cuadro II. Puntajes obtenidos en NEUROPSI.

\begin{tabular}{lcc}
\hline NEUROPSI & \multicolumn{2}{c}{ Puntajes obtenidos } \\
\hline Áreas evaluadas & Caso A & Caso B \\
\hline $\begin{array}{l}\text { Orientación temporo- } \\
\text { espacial y en persona }\end{array}$ & Normal & Normal \\
Atención y concentración & Normal & Alteración leve \\
Memoria (codificación) & Normal & Normal \\
Lenguaje & Normal & Normal \\
Lectura & Normal & Alteración \\
& Normal & Novera \\
Escritura & Normal & Normal \\
Funciones ejecutivas & Normal & Alteración leve \\
Memoria (evocación) & 118 (Normal & 106 (Normal) \\
Puntaje total & alto) & \\
&
\end{tabular}

\begin{tabular}{|lcc|}
\hline \multicolumn{2}{c}{ Cuadro III. Resultados WCST. } \\
\hline \multicolumn{2}{c|}{ Porcentajes } \\
\hline Respuestas & Caso A & Caso B \\
\hline Correctas & 80 & 66 \\
Incorrectas & 20 & 34 \\
Perseverativas & 16 & 10 \\
\hline
\end{tabular}

El objetivo de la investigación se cumplió ya que se logró caracterizar el perfil neuropsicológico de los casos evaluados.

Existen casos con epilepsia que con adecuada estimulación psicoeducativa pueden mostrar desarrollo adecuado de las capacidades cognoscitivas, como se observó en el caso A, ya que los puntajes que obtuvo indican desarrollo neuropsicológico esperado para su edad y escolaridad. Esto demuestra que no todos los pacientes epilépticos con crisis convulsivas tardías presentarán dificultades cognoscitivas después de las crisis.

Por otro lado, en el caso B los procesos de atención y memoria se vieron afectados concordando con lo reportado por Maestú et $\mathrm{al}^{19}$ acerca de que las funciones cognoscitivas más afectadas en los pacientes con epilepsia son la atención y la memoria.

También se vincula con el tipo de epilepsia que presenta, puesto que en los síndromes epilépticos idiopáticos generalizados predomina la actividad paroxística abundante generalizada que puede originar dificultades cognoscitivas. ${ }^{20}$

Además, la incapacidad para mantener la organización observada en el caso B durante su ejecución en el WISCONPC indica dificultades en la atención sostenida, debido a que ella no logra asociar los estímulos con una sola categoría, cambiándola por otra distinta tras haber completado cinco respuestas correctas consecutivas.

Otro aspecto importante fue que el caso B reportó no dormir lo suficiente, indicador de que tiene alteraciones en el ciclo sueño-vigilia, lo cual podría repercutir en su salud, ya que al disminuir el número de horas de sueño en el paciente epiléptico esto puede favorecer la aparición de crisis convulsivas. Una persona con epilepsia debe dormir lo suficiente, entre 7 y 10 horas aproximadamente y no alterar sus ciclos de sueño. ${ }^{21}$

Cabe mencionar que en las demás áreas de orientación, lenguaje, escritura y funciones ejecutivas se observaron puntajes dentro del promedio, de ahí la importancia de que las dificultades observadas en atención y memoria sean corregidas para no afectar las demás áreas de la cognición.

\section{Cuadro IV. Resultados escala Epworth.}

\begin{tabular}{lcc} 
& \multicolumn{2}{c}{ Respuestas } \\
\cline { 2 - 3 } Situaciones & Caso A & Caso B \\
\hline Sentado y leyendo & 1 & 1 \\
Viendo TV & 0 & 1 \\
Sentado en un lugar público & 0 & 2 \\
Viajar como pasajero en un vehículo & 1 & 2 \\
durante una hora & 3 & 1 \\
Acostarse a descansar por la tarde & 0 & 0 \\
Sentado conversando con alguien & 2 & 2 \\
Sentarse después del almuerzo & 0 & 3 \\
En un automóvil detenido por el tráfico & 7 & 12 \\
Total &
\end{tabular}


Las diferencias en la ejecución de ambos casos pueden explicarse por la edad de aparición de las crisis y el componente tónico-clónico de uno de los casos, además de las alteraciones de sueño antes mencionadas.

No se debe olvidar que en la evaluación clínica es importante mantener un compromiso ético y canalizar a los servicios de apoyo, así como dar sugerencias en los casos con dificultades, por mínimas que sean, para que no se afecte su desarrollo cognoscitivo. En el caso que presentó alteraciones de atención y memoria deben indicarse estrategias de corrección, así como dar a conocer la importancia de buenos hábitos de sueño, a fin de que no altere sus ciclos de sueño-vigilia. Deberá darse seguimiento a ambos casos para mantener control de sus funciones mentales.

La detección temprana y la intervención oportuna permiten la prevención de dificultades neuropsicológicas que pueden interferir en el desempeño académico de los casos con epilepsia de ausencia juvenil.

\section{CONCLUSIONES}

Las evaluaciones neuropsicológicas permitieron describir el estado actual de los procesos cognoscitivos de las participantes, así como generar posibles estrategias de intervención y estimulación cognoscitiva para las dificultades observadas en las evaluaciones.

La evaluación neuropsicológica en los casos con epilepsia es de gran importancia pues permite determinar si existen dificultades asociadas con el padecimiento.

Con las evaluaciones realizadas se mostró que existen casos con epilepsia de ausencia juvenil que tienen un desarrollo cognoscitivo adecuado.

Los dos casos evaluados mostraron ejecución normal y en uno de ellos se observaron dificultades de atención, memoria y alteraciones de sueño.

Las dificultades presentes en atención y memoria deben ser corregidas a fin de que esto no repercuta en su desempeño académico.

\section{BIBLIOGRAFÍA}

1. Organización Panamericana de la Salud. Estrategias y plan de acción sobre epilepsia. Washington: OPS, 2011; pp. 1-5.

2. Hospital General de México. Guías clínicas de diagnóstico y tratamiento, Servicio de Pediatría. Epilepsia. Hospital General de México. [Internet] 2012 [consultado el 10 de junio del 2014]. http://www.hgm.salud.gob.mx/descargas/pdf/area medica/pediatria/consulta_externa/1_epiepilep.pdf
3. Liga Internacional Contra la Epilepsia. Definición clínica práctica de la epilepsia. Epilepsia. 2014; 55: 475-482.

4. Programa Prioritario de Epilepsia. Compendio de epilepsia. México: Programa Prioritario de Epilepsia, 1997; pp. 4-8.

5. Liga Internacional Contra la Epilepsia. The etiologic classification of epilepsy. Epilepsia. 2011; 52: 1052-1057.

6. Saiz DRA, Sancho RJ. Guía Oficial de Práctica Clínica en la epilepsia. Madrid: Sociedad Española de Neurología. 2012: 17-21.

7. Sutherland J. Epilepsias: diagnóstico y tratamiento. México: El Manual Moderno, 1982; pp. 16-19.

8. Aiguabella MM et al. Pronóstico a largo plazo de la epilepsia de ausencia juvenil. Neurología. 2011; 4: 193-199.

9. Hemann B, Seidenberg M. Epilepsy and cognition. Epilepsy Curr. 2007; 7: 1-6.

10. Arnedo M, Espinosa M, Ruiz R, Sánchez-Álvarez JC. Intervención neuropsicológica en la clínica de la epilepsia. Rev Neurol. 2006; 43: 83-88.

11. Matute E, Rosselli M, Ardila A, Ostrosky-Solís F. Evaluación neuropsicológica infantil (ENI): Manual de aplicación. México: El Manual Moderno; 2007.

12. Ure J. Deterioro cognitivo en pacientes epilépticos. Rev Argent Neuropsicol. 2004; 2: 1-14.

13. Velasco R, Castro $C$. Trastornos neuropsicológicos en niños con epilepsia. Neuropsiquiatría. 2002; 65: 99-103.

14. Santín J. Sueño y epilepsia. Rev Med Clin. CONDES. 2013; 24: $480-485$.

15. Ostrosky-Solís F, Ardila A, Rosselli M. NEUROPSI: Evaluación Neuropsicológica Breve en Español. Manual e instructivo. México: Bayer de México: 11-30.

16. Guevara PMA, Hernández GM, Sanz MA, Amezcua GC. Programas computacionales para evaluar funcionalidad cognoscitiva. Guanajuato: Universidad de Guanajuato: 129-170.

17. Johns MW. A new method for measuring daytime sleepiness: the Epworth sleepiness scale. Sleep. 1991; 14: 540-545.

18. Campos-Castelló J, Campos Soler, S. Neuropsicología y epilepsia. Rev Neurol. 2004; 39: 166-177.

19. Maestú F, Martín P, Sola RG, Ortiz P. Neuropsicología y deterioro cognitivo en la epilepsia. Rev Neurol. 1999; 28: 793-798.

20. Narbona J, Crespo-Egílaz N. Trastornos de memoria y de atención en disfunciones cerebrales del niño. Rev Neurol. 2005; 40: 30-36.

21. Sánchez AJC, Galán BJ, Serrano CP. 50 respuestas sobre las crisis epilépticas y la epilepsia. Guía destinada a personas con epilepsia y a sus familiares. Barcelona: Disseny directe, 2004: 51.

Dirección para Correspondencia:

Amador Barreda Guzmán.

Manantial de San Cristóbal s/n, $2^{\circ}$ piso, Xalapa 2000, 91700 ,

Xalapa, Veracruz, México. Tel. 01228842 1700, ext. 19518.

E-mail: zS11014002@estudiantes.uv.mx 